\title{
RESEARCH
}

\section{Pharmacy Students' Knowledge and Confidence of Penicillin Allergies Following Focused Didactic Instruction and Simulation}

\author{
Wesley D. Kufel, PharmD, ${ }^{\mathrm{a}, \mathrm{b}, \mathrm{c}}$ Bruce E. Blaine, PhD, ${ }^{\mathrm{d}}$ Rachel Ruehl, PharmD, ${ }^{\mathrm{e}}$ Lisa M. Avery, PharmD ${ }^{\mathrm{f}, \mathrm{g}}$ \\ ${ }^{\text {a } B i n g h a m t o n}$ University, School of Pharmacy and Pharmaceutical Sciences, Binghamton, New York \\ ${ }^{\mathrm{b}}$ State University of New York, Upstate Medical University, Syracuse, New York \\ ${ }^{\mathrm{c}}$ State University of New York, Upstate University Hospital, Syracuse, New York \\ ${ }^{\mathrm{d}}$ Saint John Fisher College, Rochester, New York \\ ${ }^{\mathrm{e}}$ Good Samaritan TriHealth Hospital, Cincinnati, Ohio \\ ${ }^{\mathrm{f}}$ Saint John Fisher College, Wegmans School of Pharmacy, Rochester, New York \\ ${ }^{g}$ Saint Joseph's Health, Syracuse, New York
}

Corresponding Author: Wesley D. Kufel, Binghamton University, School of Pharmacy and Pharmaceutical Sciences, PO Box 6000, Binghamton, NY 13902-6000. Tel: 607-777-5849. Email: wkufel@binghamton.edu

Submitted April 13, 2021; accepted June 30, 2021; ePublished July 2021

Objective. To evaluate pharmacy students' knowledge and confidence regarding penicillin allergy assessment and skin testing (PAAST) before and after a focused didactic instruction and simulation (FDIS).

Methods. A multicenter, quasi-experimental, cross-sectional survey study was performed among pharmacy students before and after FDIS on PAAST at two schools of pharmacy. The FDIS on PAAST consisted of an infectious disease faculty-led seminar, student-led penicillin allergy counseling interviews, penicillin skin testing simulation, and case studies to assess penicillin allergy scenarios and management. An anonymous, voluntary, electronic survey was distributed to students $(n=159)$ before and after the FDIS. The pre- and post-intervention survey contained ten PAAST knowledge-based questions and multi-step, 5-point Likert scale statements related to confidence of PAAST. The postintervention survey also evaluated students' perceptions of the FDIS on PAAST. Descriptive statistics were performed, and the Student $t$-test was used to compare pre- and post-intervention responses.

Results. One-hundred and forty-three surveys were completed resulting in a survey response rate of $90 \%$. PAAST knowledge scores (mean \pm SD) increased overall following the FDIS on PAAST (6.67 \pm 1.51 vs. 7.81 \pm 1.39$)$. Knowledge scores increased considerably for questions related to penicillin allergy consequences, cross-reactivity, and correct steps of PAAST. Pharmacy students' PAAST confidence scores (mean \pm SD) also improved following the interactive instruction and simulation $(2.30 \pm 0.70$ vs. $3.22 \pm 0.67)$ with considerable confidence increases in penicillin skin testing. Pharmacy students' perceptions of the FDIS on PAAST were also positive overall.

Conclusion. Pharmacy students' knowledge and confidence of PAAST improved following FDIS. This may be an effective strategy to implement PAAST education during pharmacy school.

Keywords: penicillin allergy, simulation, penicillin skin test, pharmacy students, antimicrobial stewardship

\section{INTRODUCTION}

Penicillin allergy is the most commonly reported antibiotic allergy with approximately $10 \%$ of the United States population being labeled as penicillin allergic; however, approximately only 1 in 10 of these individuals are truly allergic. ${ }^{1-3}$ Documentation of inaccurate penicillin allergies often leads to the use of alternative antibiotics that are more broad-spectrum or less effective. ${ }^{4}$ This practice has been associated with several untoward consequences including Clostridioides difficile-associated diarrhea, increased development of resistant bacteria, increased medical costs, increased surgical site infections, and poorer clinical outcomes. ${ }^{5-12}$ Given the complications of penicillin allergies, antimicrobial stewardship programs (ASP) can be a critical resource to encourage penicillin allergy assessment and skin testing (PAAST) to identify and manage individuals who are not truly penicillin allergic. ${ }^{13-15}$

Pharmacists are well positioned to evaluate, manage, and de-label penicillin allergies as part of ASP initiatives. ${ }^{16-}$

${ }^{18}$ Pharmacists are frequently relied upon for their drug expertise within ASPs, ${ }^{19}$ and are involved in several activities related to PAAST in the inpatient setting such as leading penicillin allergy interviews, performing penicillin skin testing, 
evaluating cross-reactivity potential among beta-lactam antibiotics, and providing other types of allergy evaluations or challenges. ${ }^{20-25}$ Pharmacist opportunities and involvement in PAAST are also expanding to outpatient settings. ${ }^{26}$

Given the significant impact pharmacists can have in PAAST, appropriate education is essential. ${ }^{24,26}$ Schools of pharmacy should educate and train pharmacy students about PAAST as they may frequently be involved in such practices as future pharmacists. However, there are currently no reports of PAAST training in the PharmD curricula or other healthcare disciplines to our knowledge. Thus, we sought to evaluate the impact of a focused didactic instruction and simulation (FDIS) class on PAAST on pharmacy students' knowledge and confidence.

\section{METHODS}

This was a multicenter, quasi-experimental, cross-sectional survey study among pharmacy students before and after the FDIS on PAAST at Binghamton University School of Pharmacy and Pharmaceutical Sciences in Binghamton, New York, and Saint John Fisher College Wegmans School of Pharmacy in Rochester, New York. Pharmacy students were included in survey distribution if they attended and participated in the FDIS on PAAST. Pharmacy students were excluded if they did not complete or had an incomplete pre- and/or post-survey response.

This study was deemed exempt by the Institutional Review Boards at Binghamton University and Saint John Fisher College. A survey invitation letter with the appropriate informed consent information was attached to the survey instrument and distributed electronically via email. By selecting to enter the survey, the respondent agreed to participate.

The required 3-hour skills lab course is run concurrently with the infectious diseases (ID) pharmacotherapeutics course at both schools of pharmacy. The FDIS on PAAST occurred in the skills lab course during February 2020 of the second and third professional year at Binghamton University School of Pharmacy and Pharmaceutical Sciences and Saint John Fisher College Wegmans School of Pharmacy, respectively. Allergy basics were taught during the ID pharmacotherapeutics course prior to this intervention, but within the same week of the skills lab course. Instruction for these allergy topics was general and briefly introduced compared to this intervention's structure and content delivery.

During this session, pharmacy students were exposed to several concepts and areas within PAAST. These included a brief, 30-minute lecture on PAAST delivered by an ID pharmacist faculty member, student-led penicillin allergy interviews and activities, a simulated scratch test component of penicillin skin testing, and case studies to assess penicillin allergy scenarios and management strategies. The brief lecture on PAAST provided education on various topics related to penicillin allergies including penicillin allergy epidemiology, Centers for Disease Control and Prevention penicillin allergy statistics, ${ }^{27}$ penicillin allergy consequences, types of allergic reactions, penicillin allergy interview questions, cross-reactivity between beta-lactam antibiotics, and allergy reconciliation strategies including penicillin skin testing, direct/graded challenges, and desensitization (induction of drug tolerance). ID pharmacist faculty used the same presentation materials at both schools of pharmacy to optimize consistency in content delivery. The student-led penicillin allergy interviews and activities facilitated appropriate questions to ask patients who are labeled with a penicillin allergy. The ID pharmacist faculty member also provided a brief simulation session on the appropriate steps to complete a penicillin skin test, and then pharmacy students performed the scratch component of the penicillin skin test on fellow students as part of this activity. Lastly, pharmacy students worked in groups to complete four graded case studies to evaluate penicillin allergy management strategies with the goal of providing the correct reconciliation strategy and supporting rationale.

A 25-item pre-intervention survey and a 31-item post-intervention survey were designed to evaluate pharmacy students' knowledge and confidence of PAAST before and after FDIS. The survey was anonymous and students were not required to complete this, but participation was encouraged. The survey was developed using the expert opinion of the ID pharmacist faculty members. Qualtrics (Qualtrics, Inc., Provo, UT, USA) was used to design and collect survey responses.

The pre-intervention survey included 10 knowledge-based PAAST questions including penicillin allergy statistics, penicillin allergy consequences, types of allergic reactions, cross-reactivity between beta-lactam antibiotics, and penicillin allergy management scenarios. The pre-intervention survey also included 15 confidence statements for various aspects of PAAST measured on a 0 (strongly disagree) to 4 (strongly agree) scale. The post-intervention survey included the same knowledge questions and confidence statements as those on the pre-intervention survey as well as six additional items that measured students' perceptions of the FDIS on PAAST, also measured on a 0 (strongly disagree) to 4 (strongly agree) scale. Reliability analysis of the pre- and post-intervention scale score demonstrated that the scale had excellent internal consistency with a Cronbach's alpha value of 0.92 and 0.97 , respectively.

The survey instrument was pilot tested by an ID pharmacist and fourth year pharmacy student. Feedback was then incorporated before dissemination to ensure validity, logistical integrity, and question clarity. The pre-intervention survey was distributed electronically via email to pharmacy students shortly prior to the start of class and subsequently closed before instruction began. The post-intervention survey was distributed electronically via email to pharmacy students at the 
conclusion of class and was left open for 48 hours after. No reminder emails were sent given the relatively short time the survey remained open.

Survey responses were collected via Qualtrics survey software and statistical analyses were performed using R (R Foundation for Statistical Computing, Vienna, Austria). Survey responses were matched based on unique, anonymous survey codes to align pre- and post-survey responses. Descriptive statistics were performed to characterize survey responses. Comparisons of continuous data within the survey were performed using a Student $t$-test. All statistical tests were 2-tailed, and a p-value less than 0.05 was considered to indicate a statistically significant difference.

\section{RESULTS}

One hundred and forty-three out of 159 pharmacy students completed the survey resulting in a response rate of $90 \%$. Thus, 16 out of 159 pharmacy students were excluded because of incomplete or unsubmitted survey responses. Seventy-seven (54\%) and 67 (46\%) pharmacy students responded from Binghamton University School of Pharmacy and Pharmaceutical Sciences and Saint John Fisher College Wegmans School of Pharmacy, respectively. More respondents were female (63\%) compared to male (37\%).

Overall mean knowledge scores on ten PAAST-related questions significantly improved from pre-intervention to post-intervention $(6.67 \pm 1.51$ versus $7.81 \pm 1.39, p<.001)$. Table 1 displays the pre- and post-survey PAAST knowledge scores by item. All questions demonstrated statistically significant improvement following FDIS. Knowledge scores increased by an average of one point overall following the intervention $(p<.001)$. Furthermore, 59\% of pharmacy students' knowledge scores improved with $37 \%$ of them improving by $\geq 2$ points.

Overall mean confidence agreement with PAAST statements using a 5-point (0-4) Likert scale significantly improved from pre-intervention to post-intervention $(2.3 \pm 0.7$ versus $3.22 \pm 0.67, p<.001)$. Table 2 shows the mean improvement in confidence agreement scores for PAAST following FDIS. Pharmacy students' confidence increased significantly in all PAAST areas assessed. Confidence scores increased the most for penicillin skin testing, specifically with the steps to perform penicillin skin testing, the scratch test component, the intradermal component, and management of potential adverse reactions.

Table 3 displays pharmacy students' perceptions of the FDIS on PAAST. Pharmacy students' perceptions of the class were positive overall. On a 0-to-4-point scale, the mean agreement for each statement was above 3.4. Based on anecdotal information, pharmacy students were interested in having additional FDIS sessions in the future, and believed that participation will better prepare them for their clinical rotations and future practice as a pharmacist. They found the class enjoyable and that it generally improved their knowledge and comfort level for evaluation and management of PAAST.

\section{DISCUSSION}

Given the importance of pharmacists as drug experts and educators for ASP, pharmacy graduates involved in antimicrobial stewardship roles will likely engage in PAAST in both inpatient and outpatient settings. ${ }^{19,24,26}$ Postgraduate training opportunities for pharmacists specializing in ID are available, but a limited number of programs currently exist. ${ }^{28}$ A 15-hour continuing education certificate program is offered by the University of South Carolina College of Pharmacy to specifically train and certify pharmacists and other clinicians in PAAST, which also includes a four-hour live component with hands-on teaching and practice with penicillin skin testing. ${ }^{29}$ Given these limited opportunities for pharmacy graduates, PAAST education and training should be considered to begin in schools of pharmacy.

We describe the implementation of FDIS on PAAST at two schools of pharmacy during a skills lab course. This interactive class was designed to teach and apply key components of the PAAST process including, but not limited to basic penicillin allergy knowledge, penicillin allergy statistics, beta-lactam antibiotic cross-reactivity, types of allergic reactions, penicillin allergy interview assessments, penicillin skin testing, and penicillin allergy management. Our goal was to provide pharmacy students with the necessary education and training to evaluate and manage penicillin allergies. We observed a statistically significant increase in overall knowledge scores on a ten-question PAAST assessment, and also on specific items, most notably those involved with penicillin allergy consequences, cross-reactivity, and correct steps of PAAST. We also observed a statistically significant increase in pharmacy students' confidence related to PAAST. Although pre-intervention to post-intervention confidence increased significantly on all items, the confidence boosts were particularly large on items related to penicillin skin testing. Furthermore, students generally enjoyed this interactive focused class on PAAST and found this educational initiative useful to their practice as a future pharmacist.

Current literature outlines the success of simulation experiences for various clinical pharmacy activities including medication reconciliation, patient counseling, and pharmacokinetic dosing. ${ }^{30-32}$ To our knowledge, this is the first report to describe and evaluate PAAST education and simulation training in the PharmD curricula. It is unknown if and to what 
extent PAAST education and training exists in PharmD programs. While allergies and drug hypersensitivities would be expected in the PharmD curricula, ${ }^{33}$ it is unclear if this also includes PAAST. Kufel and colleagues evaluated the inclusion and extent of antimicrobial stewardship education among schools of pharmacy in the United States, and identified that only approximately $68 \%$ of respondents included antimicrobial stewardship in their required didactic curricula. However, it is still unknown if this education specifically included PAAST. As such, we hope our experience will further encourage pharmacy educators to consider prioritizing PAAST education and training within the PharmD curricula.

One of the strengths of our study was the high survey response rate of $90 \%$ to potentially minimize the risk of selection bias. Nevertheless, our study is not without important limitations to consider. First, we surveyed pharmacy students soon after the FDIS on PAAST was delivered. Therefore, it is unclear if students will have retained the content and application upon graduation for future practice. Future studies should perform follow-up assessments to evaluate if the content and skills learned were retained. Second, our surveyed population is only representative of pharmacy students from two schools of pharmacy and may not be generalizable to pharmacy students at other schools of pharmacy. Third, our instruction was delivered by two different ID pharmacist faculty members at each school of pharmacy, and thus, the content delivery may have varied. However, we utilized the same lecture content and activities for teaching to be as consistent as possible. Lastly, given the limited number of knowledge-based questions asked, there is a potential wide range for significance where small changes in the number of correct or incorrect responses could impact results.

\section{CONCLUSIONS}

Our study demonstrated that delivery of a FDIS on PAAST to pharmacy students led to significant improvements in knowledge scores and confidence for performing PAAST. Pharmacy students also described that they enjoyed this educational experience and desired more FDIS in other areas. Future evaluations of PAAST education and training within the pharmacy school curricula are needed since it is unclear if and to what extent such exists. We hope our structure and delivery can serve as a model for schools of pharmacy to increase PAAST education for pharmacy students.

\section{ACKNOWLEDGMENTS}

The authors would like to acknowledge ALK-Abello, Inc. for providing the penicillin skin testing supplies for classroom instruction. No financial support was used for this research endeavor. Author Disclosures: Wesley D. Kufel has received research grants from Merck and Melinta Therapeutics, and served on the advisory board for Theratechnologies, Inc. Lisa M. Avery's husband is an employee of Merck. Rachel Ruehl was an employee of ALK at the time of this study. All other others have nothing to disclose. This study was carried out as part of our routine work.

\section{REFERENCES}

1. $\quad$ Drug allergy: an updated practice parameter. Ann Allergy Asthma Immunol. 2010;105(4):259-273.

2. Macy E. Penicillin and beta-lactam allergy: epidemiology and diagnosis. Curr Allergy Asthma Rep. 2014;14(11):476.

3. Shenoy ES, Macy E, Rowe T, Blumenthal KG. Evaluation and Management of Penicillin Allergy: A Review. JAMA. 2019;321(2):188-199.

4. $\quad \mathrm{Wu} \mathrm{JH}$, Langford BJ, Schwartz KL, et al. Potential Negative Effects of Antimicrobial Allergy Labelling on Patient Care: A Systematic Review. Can J Hosp Pharm. 2018;71(1):29-35.

5. Charneski L, Deshpande G, Smith SW. Impact of an antimicrobial allergy label in the medical record on clinical outcomes in hospitalized patients. Pharmacotherapy. 2011;31(8):742-747.

6. Al-Hasan MN, Acker EC, Kohn JE, Bookstaver PB, Justo JA. Impact of Penicillin Allergy on Empirical Carbapenem Use in Gram-Negative Bloodstream Infections: An Antimicrobial Stewardship Opportunity. Pharmacotherapy. 2018;38(1):42-50.

7. Blumenthal KG, Lu N, Zhang Y, Li Y, Walensky RP, Choi HK. Risk of meticillin resistant Staphylococcus aureus and Clostridium difficile in patients with a documented penicillin allergy: population based matched cohort study. BMJ. 2018;361:k2400.

8. Trubiano JA, Chen C, Cheng AC, Grayson ML, Slavin MA, Thursky KA. Antimicrobial allergy 'labels' drive inappropriate antimicrobial prescribing: lessons for stewardship. J Antimicrob Chemother. 2016;71(6):1715-1722.

9. Su T, Broekhuizen BDL, Verheij TJM, Rockmann H. The impact of penicillin allergy labels on antibiotic and health care use in primary care: a retrospective cohort study. Clin Transl Allergy. 2017;7:18.

10. Sacco KA, Bates A, Brigham TJ, Imam JS, Burton MC. Clinical outcomes following inpatient penicillin allergy testing: A systematic review and meta-analysis. Allergy. 2017;72(9):1288-1296. 
11. Blumenthal KG, Ryan EE, Li Y, Lee H, Kuhlen JL, Shenoy ES. The Impact of a Reported Penicillin Allergy on Surgical Site Infection Risk. Clin Infect Dis. 2018;66(3):329-336.

12. Sousa-Pinto B, Blumenthal KG, Macy E, et al. Penicillin allergy testing is cost-saving: An economic evaluation study. Clin Infect Dis. 2021;72(6):924-938.

13. Staicu ML, Vyles D, Shenoy ES, et al. Penicillin Allergy Delabeling: A Multidisciplinary Opportunity. J Allergy Clin Immunol Pract. 2020;8(9):2858-2868.e2816.

14. Barlam TF, Cosgrove SE, Abbo LM, et al. Executive Summary: Implementing an Antibiotic Stewardship Program: Guidelines by the Infectious Diseases Society of America and the Society for Healthcare Epidemiology of America. Clin Infect Dis. 2016;62(10):1197-1202.

15. Blumenthal KG, Phillips EJ. Positioning Drug Allergy Delabeling as a Critical Tool for Precision Medicine, Quality Improvement, and Public Health. J Allergy Clin Immunol Pract. 2020;8(9):2916-2919.

16. Mancini CM, Fu X, Zhang Y, et al. Penicillin Allergy Evaluation Access: A National Survey. Clin Infect Dis. 2020;71(11):2972-2975.

17. Mancini CM, Kuper K, Schulz LT, et al. Reply to Bland and Jones. Clin Infect Dis. 2020; Epub ahead of print.

18. Bland CM, Jones BM. Pharmacists Filling the Gap Within Penicillin Allergy Assessment and Skin Testing. Clin Infect Dis. 2020; Epub ahead of print.

19. Centers for Diseases Control and Prevention. Hospital antibiotic stewardship. National Healthcare Safety Network. https://arpsp.cdc.gov/profile/stewardship. Accessed March 15, 2021.

20. Sigona NS, Steele JM, Miller CD. Impact of a pharmacist-driven beta-lactam allergy interview on inpatient antimicrobial therapy: A pilot project. J Am Pharm Assoc. 2016;56(6):665-669.

21. Mann KL, Wu JY, Shah SS. Implementation of a Pharmacist-Driven Detailed Penicillin Allergy Interview. Ann Pharmacother. 2020;54(4):364-370.

22. Englert E, Weeks A. Pharmacist-driven penicillin skin testing service for adults prescribed nonpreferred antibiotics in a community hospital. Am J Health-Syst Pharm. 2019;76(24):2060-2069.

23. Justo JA, Kufel WD, Avery L, Bookstaver PB. Penicillin Allergy Skin Testing in the Inpatient Setting. Pharmacy (Basel). 2019;7(3).

24. Bland CM, Bookstaver PB, Griffith NC, et al. A practical guide for pharmacists to successfully implement penicillin allergy skin testing. Am J Health-Syst Pharm. 2019;76(3):136-147.

25. Griffith NC, Justo JA, Winders HR, et al. Regulatory approval, implementation, and brief assessment of a pharmacist- and pharmacy trainee-administered penicillin allergy assessment and skin testing program. $J$ Am Coll Clin Pharm. 2020;3:1269-1279.

26. Kufel WD, Justo JA, Bookstaver PB, Avery LM. Penicillin Allergy Assessment and Skin Testing in the Outpatient Setting. Pharmacy (Basel). 2019;7(3).

27. Centers for Disease Control and Prevention. Is it Really a Penicillin Allergy? https://www.cdc.gov/antibioticuse/community/pdfs/penicillin-factsheet.pdf. Accessed March 15, 2021.

28. Gauthier TP, Worley M, Laboy V, et al. Clinical infectious diseases pharmacists in the United States: a problem of both supply and demand. Clin Infect Dis. 2015;60(5):826-827.

29. University of South Carolina College of Pharmacy. Penicillin Allergy Assessment \& Skin Testing (PAAST) Certificate Program. 2020.

https://www.sc.edu/study/colleges_schools/pharmacy/centers/penicillin_allergy_skin_testing_certificate_program I. Accessed March 5, 2021.

30. Bajis D, Chaar B, Basheti IA, Moles R. Pharmacy students' medication history taking competency: Simulation and feedback learning intervention. Curr Pharm Teach Learn. 2019;11(10):1002-1015.

31. Mak V, Sandhu AK, Krishnan S. Using Simulation to Teach Methods for Improving Patient Literacy about Medicines. Pharmacy (Basel). 2020;8(4).

32. Cropp CD, Beall J, Buckner E, Wallis F, Barron A. Interprofessional Pharmacokinetics Simulation: Pharmacy and Nursing Students' Perceptions. Pharmacy (Basel). 2018;6(3).

33. Flannery AH, Soric MM, Benavides S, et al. 2019 Update to the American College of Clinical Pharmacy Pharmacotherapy Didactic Curriculum Toolkit. J Am Coll Clin Pharm. 2020;3:455-464. 
Table 1. Pre- and Post-Intervention PAAST Knowledge Scores by Item ( $\mathrm{n}=143)$.

Pre-

\section{Question}

Number

1 Symptoms of IgE-mediated allergic reactions

Intervention

(Percentage

$2 \quad$ Percentage of individuals labeled as penicillin allergic

Correct)

3 Percentage of individuals truly allergic to penicillin

Percentage of individuals with IgE-mediated allergic

4 reactions to penicillin who lose sensitivity at ten years

5 Untoward consequences of labeled penicillin allergies

Beta-lactam antibiotic cross-reactivity based on R-1

6

side chain

$94 \%$

$73 \%$

$65 \%$

Post-

Intervention

(Percentage

Correct)

P.

$98 \%$

value $^{\text {a }}$

$<.001$

$87 \% \quad<.001$

$71 \%-<.001$

$82 \% \quad 97 \% \quad<.001$

$42 \% \quad 82 \% \quad<.001$

Rashes in children with Epstein Barr virus infections

7 who receive penicillin-type antibiotics

$66 \% \quad 88 \% \quad<.001$

Recommend penicillin skin testing for an appropriate

8 patient

9 Graded challenge versus desensitization

$96 \%$
$29 \%$
$27 \%$
$80 \%$

$88 \%<.001$

10 Identifying the correct steps in the PAAST process

$80 \%$

$99 \%$

$<.001$

${ }^{a}$ p-values are from t-test analyses of pre- to post-intervention improvement.

Abbreviations: Immunoglobulin E, IgE; PAAST, penicillin allergy assessment and skin testing.

Table 2. Mean Pre- to Post-Intervention Improvement in Confidence Agreement Scores for PAAST 
Following the Focused Didactic Instruction and Simulation Class ( $\mathrm{n}=143)$.

\begin{tabular}{|c|c|c|}
\hline $\begin{array}{l}\text { Question } \\
\text { number }\end{array}$ & PAAST category or topic & $\begin{array}{l}\text { Mean improvement }{ }^{\mathrm{a}} \\
(95 \% \text { CI })\end{array}$ \\
\hline 1 & Penicillin allergy statistics and epidemiology & $0.62(0.46-0.79)$ \\
\hline 2 & Untoward consequences of penicillin allergies & $0.45(0.29-0.62)$ \\
\hline 3 & Cross-reactivity between beta-lactam antibiotics & $0.79(0.59-0.99)$ \\
\hline 4 & IgE-mediated allergic reactions & $0.38(0.22-0.54)$ \\
\hline 5 & Ability to perform a penicillin allergy interview & $0.86(0.67-1.05)$ \\
\hline 6 & Provide penicillin allergy education to patients & $0.67(0.51-0.84)$ \\
\hline 7 & Provide penicillin allergy education to clinicians & $0.93(0.74-1.12)$ \\
\hline 8 & $\begin{array}{l}\text { Differentiate desensitization versus graded challenges } \\
\text { Recommend penicillin skin testing for an appropriate }\end{array}$ & $1.05(0.84-1.25)$ \\
\hline 9 & patient & $0.96(0.75-1.17)$ \\
\hline 10 & Correct steps to perform a complete penicillin skin test & $1.45(1.24-1.65)$ \\
\hline 11 & Correct steps to perform the scratch test component & $1.78(1.56-1.99)$ \\
\hline 12 & $\begin{array}{l}\text { Correct steps to perform the intradermal component } \\
\text { Management of potential adverse reactions during a }\end{array}$ & $1.42(1.20-1.64)$ \\
\hline 13 & penicillin skin test & $1.41(1.20-1.61)$ \\
\hline 14 & $\begin{array}{l}\text { Interpretation of penicillin skin test results } \\
\text { Delabeling the penicillin allergy following a negative }\end{array}$ & $0.67(0.45-0.89)$ \\
\hline 15 & $\begin{array}{l}\text { penicillin skin test } \\
\end{array}$ & $0.45(0.67-1.24)$ \\
\hline
\end{tabular}


Table 3. Post-Intervention Perceptions of the Focused Didactic Instruction and Simulation Class on PAAST $(\mathrm{n}=143)$.

\begin{tabular}{ccc}
\hline Item & Mean (SD) \\
\hline
\end{tabular}

I am interested in having more focused instruction and simulation sessions for infectious disease education in the future.

I feel that participation in this penicillin allergy instruction and simulation will better prepare me for my clinical rotations.

I feel more comfortable performing an evaluation and providing recommendations for a patient with a penicillin allergy following penicillin allergy instruction and simulation.

I learned content in the penicillin allergy instruction and simulation that will be useful in my future practice as a pharmacist.

Penicillin allergy instruction and simulation was an enjoyable active learning experience.

Penicillin allergy instruction and simulation improved my knowledge of penicillin allergies.

$3.55(0.72)$

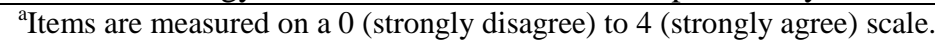

$3.47(0.79)$

Abbreviations: PAAST, penicillin allergy assessment and skin testing; SD, standard deviation. 